\title{
ОБ ОДНОМ МЕТОДЕ ПОИСКА СИММЕТРИЧНЫХ БИЛИНЕЙНЫХ АЛГОРИТМОВ УМНОЖЕНИЯ МАТРИЦ
}

Чокаев Б. В.

ГГНТУ им. акад. М.Д. Миллионщикова, Чеченский государственный университет,

г. Грозный

Известно, что билинейный алгоритм для умножения матрии $3 \times 3$ можно задавать с помощью упорядоченных троек $3 \times 3$-матриц $A, B, C$. Широко изучаемыми являются алгоритмы, обладающие различными симметриями. Актуальной задачей является разработка алгоритмов, обладающих различными симметриями. В данной статье представлены два алгоритма сложности 25, обладающие определенными симметриями. Показано, что найденные алгоритмы являются различными и новыми.

Ключевые слова: умножение матриц, алгоритм Штрассена, билинейная сложность.

Одной из центральных областей алгебраической теории сложности является сложность умножения в алгебрах. Задача сложности умножения в алгебре заключается в том, чтобы построить алгоритм, который для любых двух элементов алгебры вычислял бы их произведение, и имел бы наименьшую сложность. При этом под сложностью алгоритма могут подразумеваться различные понятия. Например, можно учитывать все арифметические операции над полем, которые требуются для вычисления произведения в алгебре. Возникающая сложность называется арифметической (тотальной) сложностью. Другой способ определения сложности - учитывать только так называемые существенные умножения, то есть такие операции умножения, оба операнда в которых зависят от входных переменных. В этом случае возникают понятия билинейного алгоритма и билинейной сложности (или ранга). Если рассматривать последовательность билинейных алгоритмов, сходящаяся к билинейному алгоритму для умножения в данной алгебре, то возникают более общие понятия - приближенного билинейного алгоритма и граничного ранга алгебры.

Во многих практических алгоритмах (в частности, в криптографии и в комбинаторных алгоритмах) активно используются различные алгебраические операции. В частности, операция умножения матриц лежит в основе сложности не только большинства задач линейной алгебры, но и множества комбинаторных задач. Например, для получения быстрых алгоритмов для задач построения замыкания графа и нахождения всех кратчайших путей в графе достаточно иметь быстрый алгоритм умножения матриц. 
Кроме того, задача нахождения ранга билинейного отображения, в частности ранга умножения в алгебре, лежит в основе многих прикладных задач из различных областей науки. Примерами могут служить следующие задачи: интерпретация магнитно-резонансной томографии в медицине, интерпретация магнитотеллурических данных для одномерных и двумерных региональных структур в геофизике, определение соединений в растворе с использованием флуоресцентной спектроскопии в химии, определение местоположения источника радиосигнала по данным из нескольких приемников в радиотехнике.

Задача умножения матрицы размера $\mathrm{m}$ на $\mathrm{n}$ на матрицу размера $\mathrm{n}$ на $\mathrm{p}-$ это задача вычисления системы из $\mathrm{mp}$ билинейных форм. Билинейным алгоритмом сложности $r$ для этой задачи называется следующая последовательность шагов: 1) вычисление $2 \mathrm{r}$ линейных форм от входных переменных; 2) вычисление $\mathrm{r}$ их попарных произведений; 3) вычисление каждой из $\mathrm{mp}$ искомых билинейных форм в виде линейной комбинации найденных билинейных форм. Билинейной сложностью этой задачи называется наименьшее $\mathrm{r}$ среди всех билинейных алгоритмов для нее, она обозначается $\mathrm{rk}(\mathrm{m}, \mathrm{n}, \mathrm{p})$.

Исследование билинейной сложности умножения матриц даже малых размеров является непростой задачей - на сегодняшний день установлены значения билинейной сложности только для трех наборов значений параметров $^{2} \mathrm{~m}, \mathrm{n}$ и $\mathrm{p}: \operatorname{rk}(2,2,2)=7, \operatorname{rk}(2,2,3)=11, \operatorname{rk}(2,2,4)=14$. Для умножения матриц $3 \times 3$ еще в 1976 г. Дж. Ладерманом был найден алгоритм сложности 23, но с тех пор понизить эту оценку не удалось (текущая нижняя оценка равна 19). Тем не менее, периодически появляются статьи, посвященные билинейным алгоритмам умножения матриц $3 \times 3$. Статья [1] посвящена исследованию симметрий известных алгоритмов. В работе [2] приводится не известный ранее алгоритм сложности 23, симметричный относительно действия циклических групп порядков 3 и 4. В статье [3] показывается, как аналитически строить алгоритм сложности 25, симметричный относительно группы перестановок порядка 4 и имеющий некоторую дополнительную симметрию.

Симметричные алгоритмы для задач малой размерности представляют интерес по нескольким причинам. Во-первых, они являются более простыми для понимания, чем билинейные алгоритмы общего вида. Во-вторых, для того чтобы их задать, достаточно обозначить некоторую часть алгоритма и определить правила порождения остальных частей при помощи действия группы. В-третьих, такие алгоритмы могут помочь понять, как устроены оптимальные билинейные алгоритмы для общей задачи (произвольной размерности), так как в симметричных алгоритмах легче заметить закономерность. К тому же, так как сама операция умножения как билинейное отображение обладает рядом симметрий, то, вероятно, оптимальный алгоритм для него также будет обладать этими симметриями.

\footnotetext{
${ }^{2} \mathrm{C}$ учетом того, что $\mathrm{rk}(\mathrm{m}, \mathrm{n}, \mathrm{p})$ не меняется при любой перестановке параметров $\mathrm{m}, \mathrm{n}$ и p, а также, не считая тривиальных случаев, когда один или несколько параметров из $\mathrm{m}, \mathrm{n}$ и p равны 1 (в этих случаях rk (m,n,p)=mnp).
} 
Данная работа посвящена методу поиска симметричных (в некотором смысле) билинейных алгоритмов умножения матриц $3 \times 3$ путем численного решения системы уравнений.

Можно легко показать, что существование билинейного алгоритма сложности $\mathrm{r}$ для задачи умножения матрицы размера $\mathrm{m}$ на $\mathrm{n}$ на матрицу размера $\mathrm{n}$ на $\mathrm{p}$ эквивалентно существованию $\mathrm{r}(\mathrm{mn}+\mathrm{np}+\mathrm{pm})$ чисел, таких, что выполнена система из nmpnmp уравнений.

Алгоритм умножения матриц $3 \times 3$ сложности $\mathrm{r}$ представляет собой решение системы из 729 уравнений с $27 \mathrm{r}$ неизвестными $(\mathrm{m}=\mathrm{n}=\mathrm{p}=3)$. То есть алгоритм сложности $\mathrm{r}$ задается с помощью $\mathrm{r}$ упорядоченных троек матриц $\mathrm{A}, \mathrm{B}$, C размера 3х3. Под симметричным алгоритмом в данной работе будем понимать билинейный алгоритм, обладающий двумя свойствами ${ }^{3}:$ 1) в алгоритме присутствует тройка единичных матриц; 2) если в алгоритме присутствует тройка $\mathrm{A}, \mathrm{B}, \mathrm{C}$, то в нем также присутствуют две другие тройки $\mathrm{B}, \mathrm{C}, \mathrm{A}$ и С, А, B.

В данной работе проводился поиск симметричного алгоритма сложности ${ }^{4}$ $\mathrm{r}=19$ или $\mathrm{r}=22$. Для симметричного алгоритма система уравнений преобразуется в систему, в которой число неизвестных равно 9(r-1), а число различных уравнений - 249 (часть переменных и уравнений отождествляется между собой в силу свойства 2 алгоритма). Для решения этой системы использовался метод Монте-Карло, примененный к нахождению глобального минимума функционала невязки. Расчеты проводились на суперкомпьютере IBM BlueGene/P, установленном на факультете ВМК МГУ.

В первом случае минимизировался функционал, соответствующий системе с $\mathrm{r}=22$ и числом переменных $9(\mathrm{r}-1)=189$. В найденном наборе значений переменных только одно уравнение из 249 давало существенный вклад в невязку, остальные уравнения давали вклад, близкий к нулю. Во втором случае минимизировался функционал, соответствующий системе с $\mathrm{r}=19$ и числом переменных 9(r-1)=162. Найденная последовательность значений переменных была такова, что три уравнения из 249 давали существенный вклад в невязку, остальные - вклад, близкий к нулю. В обоих этих случаях визуальный анализ найденного решения позволил его преобразовать в симметричный билинейный алгоритм сложности 25 с коэффициентами из множества $\{0,1,-1,0.5,-0.5,2 / 3$, $2 / 3\}$.

\section{Исследование выполнено при финансовой поддержке РФФИ в рамках научного проекта 18-31-00044-мол-а.}

\footnotetext{
${ }^{3}$ Отметим, что этими свойствами обладает алгоритм Штрассена для умножения матриц 2х2. А также алгоритм для умножения матриц $3 \times 3$ сложности 25 из [3]. Кроме того, алгоритм Ладермана и алгоритм из [2] обладают следующим свойством, которое близко свойству 2: если в алгоритме присутствует тройка А, В, С, то либо в нем также присутствуют две другие тройки В, С, А и С, А, В, либо А = B = C.

4 Так как в симметричных алгоритмах $\mathrm{r}-1$ делится на 3 , a $\mathrm{r}$ заведомо расположено между 19 и 23 , то для $\mathrm{r}$ остается всего два варианта.
} 


\section{Список литературы}

1. Burichenko V.P. Symmetries of matrix multiplication algorithms// arXiv:1508.01110[cs.CC]. 2015.

2. Ballard G., Ikenmeyer C., Landsberg J.M., Ryder N. The geometry of rank decompositions of matrix multiplication in $3 \times 3$ matrices// arXiv:1610.08364. [cs.SC]. 2017.

3. Grochow J. A., Moore C. Matrix multiplication algorithms from group orbits // arXiv:1612.01527. 2016. 